\title{
El ser y la sustancia de Aristóteles ante la razón vital: las cuatro reducciones de la realidad
}

\section{Aristotle's being and substance in view of the vital reason: the four reductions of reality}

\author{
Francesco DE NigRIS
}

Universidad Francisco de Vitoria

Recibido: 31-01-2012

Aceptado: 02-04-2012

\section{Resumen}

«Hermenéutica» significa interpretar, mirar desde un punto de vista. En este estudio buscamos interpretar desde la «razón vital», el método de Ortega y Gasset y su discípulo Julián Marías, el concepto aristotélico de «sustancia». Si la vida es la realidad radical, como afirma Ortega, y la persona es su realización programática, según entiende Marías, una razón vital de la ov̉oía significa descubrir su capacidad de comprensión para con la vida humana. Encontraremos, entonces, que hay esencialmente cuatro límites que desvían de esa comprensión; mediante ellos, habremos encontrado el núcleo de importantes contradicciones del pensamiento aristotélico que han sido objeto de encendidos debates a lo largo de la historia. Nos centraremos, sobre todo, en la función del lenguaje en la concepción de la razón griega, en los supuestos interpretativos del «ser» y del «ente» como puntos de partida del pensamiento de Aristóteles.

Palabras clave: Aristoteles, sustancia, ente, razon vital, proyecto.

\section{Abstract}

«Hermeneutic» means interpretation, that is, looking from a point of view. In this study we try to interpret from the vital reason, the method of Ortega y Gasset, his and his disciple Julián Marías, the Aristotelian concept of «substance». If life is 
the radical reality, as Ortega stresses, and the person its programmatic realization, in Marías' opinion, a vital reason of the concept of ov̉oía, means to discover its capacity to understand human life. We will find, as a matter of fact, that there are four limits, essentially, that distance the philosopher from that comprehension. By them, we will find the core of the greatest contradictions of the Aristotelian thought, that has been subject to intense critics throughout its history. We will concern, especially, on the function of language in the Greek conception of reason, on the interpretations that brought to «being» and «entity» as Aristotle's thought starting point.

Keywords: Aristotle, substance, entity, vital reason, project.

En La idea de principio en Leibniz, José Ortega y Gasset afirma que el gran tema del «ser» es un problema intelectual y no puede ser un ciego punto de partida, una creencia ${ }^{1}$. En un cierto momento histórico, según Ortega, se han dado los supuestos que han permitido que hablar del ser fuera lo mismo que hablar de «la realidad», $\mathrm{y}$, consecuentemente, que todas y cada una de las realidades fueran «entes».

¿Cuáles son, según Ortega, los supuestos históricos del ser, que muestran claramente que es un modo que se ha dado en Grecia para comprender "todo lo que hay", la realidad?

El ser, nos dice el gran pensador madrileño, no es un concepto que ha sido extraído de las cosas según un proceso inductivo inspirado por la percepción sensible («por abstracción comunista», según su terminología), sino que es un concepto hecho en oposición a la nada y al devenir². Cuando vemos una cosa sensible, «Vemos su blancura y su esfericidad o cubicidad, oímos su sonoridad, tocamos su dureza, percibimos su movimiento, su aumento o disminución, etc.; pero no logramos columbrar su entidad o lo que tiene de Ente» ${ }^{3}$. Esto nos muestra claramente que «La

${ }^{1}$ Cf. Ortega y gasset, J., La idea de principio en Leibniz y la evolución de la teoría deductiva (1947), en O. C., vol.VIII, Revista de Occidente, 1965 1947, pp. 270-285); también, Apuntes sobre el pensamiento, su teurgia y su demiurgia (1940-41), en O. C., vol. V, Revista de Occidente, 1965, p. 532ss., o, de Julián Marías, su Introducción a la Filosofía (1947), en Tomo II de Obras, Revista de Occidente, cap. VIII apartado 64.

2 El Ente se formula en oposición a la Nada, dice Ortega poniendo en mayúscula ambas palabras, para sustantivar su importancia y unidad (la del Ente). Se trata, naturalmente, de una Nada en cuanto absurda afirmación del no existir de las cosas, que siempre evidentemente son a los ojos del hombre, y que, si van siendo, es porque están sometidas a la opinión y a la sensibilidad que se aleja de la intuición de su esencia, que consiste en ilimitada existencia. No hay que entender, naturalmente, el trasfondo del concepto de Nada en oposición al concepto cristiano de Creación. En éste la nada es la posibilidad de la no Creación, es el gozo agradecido del hombre que se encuentra abocado a la vida por el acto creador, libre y personal de Dios.

${ }^{3}$ Cf. La idea de principio en Leibniz, p. 228. 
idea del ser no ha sido extraída de las cosas, sino que ha sido introducida en ellas por el hombre, porque es previa al ser de cada una, y las hace posibles en cuanto entes»; por eso, sigue diciendo con originalidad, podemos prácticamente pensar que la deducción transcendental kantiana ya fue hecha por Aristóteles, pues la percepción sensible se organiza a priori en vista de un concepto, el Ente, que intelectualmente la determina ${ }^{4}$. Para Aristóteles y a los escolásticos, entonces, predicar «el "ente" de todo lo habible, les parece que se trata simplemente de una abstracción comunista practicada sobre todas las cosas sensibles que llegan a su natural extremismo. Ente será lo superlativamente común, lo comunísimo» ${ }^{5}$.

Una consecuencia fundamental que esto implica también para el pensamiento de Aristóteles, es que la $\alpha \tilde{i} \sigma \theta \eta \sigma i \varsigma^{6}$ sea, al final y al cabo, sensibilidad inteligente en vista del ente o, también, mejor dicho, interpretación: aquella que orienta los sentidos hacia el desvelamiento de las formas inmutables de la sustancia frente a sus accidentes materiales.

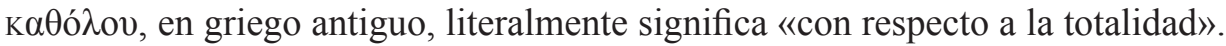
Kotvóv es lo universal en el sentido de lo que es común a distintos elementos, lo que vale para una comunidad. Y una comunidad de elementos llega a ser una totalidad (ő $\lambda$ ov) de sentido, lógica u ontológica, dependiendo del lazo de comunidad que se establece. La filosofía no es una ciencia, que intenta conocer una parcela de la realidad (química, física, biológica...), o una perspectiva más de las humanidades, que poética o novelísticamente, por ejemplo, busca comprender aspectos del mundo, sino que pretende comprender la realidad en cuanto tal, es decir, su mismo sentido. La filosofía, pues, alcanza su máximo nivel, la metafísica, cuando encuentra un principio y un fin para comprender la realidad en cuanto totalidad (ö $\lambda$ ov). Ahora bien, el principio conceptual que utiliza Parménides es la entidad. El Ente no es ni una cosa ni tampoco nos muestra cosas, sino muestra una relación entre ellas, en cuanto seres o existentes, para que las comprendamos en una comunidad de sentido que sea una totalidad. Es, podríamos decir en términos aristotélicos, el primer $\pi \rho o ̀ \varsigma$ Ěv, es decir, el principio en vista del cual la realidad cobra un sentido. Este sentido que alumbra Parménides es, como muestra Ortega, evidentemente histórico; es el de

\footnotetext{
${ }^{4}$ Ibid. p. 236.

5 Ibid. p. 228.

${ }^{6} \mathrm{La}$ intuición sensible, dice Ortega, es «la facultad fundamental de la doctrina aristotélica»; sin

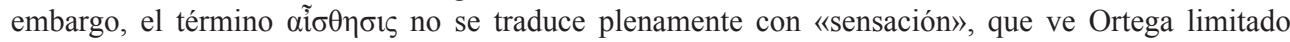
por la terminología psicológica que se imponía en su momento. Sensación es la sensación de color o del sonido, sin duda, pero también es percepción, como, por ejemplo, la percepción de una cosa singular, o es representación como la de movimiento o su reposo, figura o magnitud. La sensación es la facultad que nos conduce a los principios y que consiste, esencialmente, en discernir, en juzgar

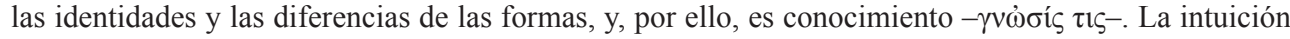
sensible, concluye Ortega, es la facultad noética en cuanto es el más puro hacerse cargo de lo que "sentimos" en el sentido pleno del término, y su resultado más inmediato es el voךtóv, el más puro inteligible. (Cf. Ibid. pp. 157-158).
} 
asegurar, frente al movimiento de la naturaleza, e, incluso, del intelecto humano que la piensa, una lógica de absoluta identidad: la existencia en sí de todo lo que hay.

Aplicando estas primeras evidencias hermenéuticas del concepto de entidad a la doctrina de Platón y de Aristóteles, sus más importantes receptores, cabe señalar lo siguiente. El pensamiento de ambos conserva, sin duda, la pretensión metafísica de comprender la totalidad de lo que hay y, por ello, su sentido, pero estableciendo, cada uno, un principio de comprensión que trasciende la evidencia de la mera existencia y busca alcanzar la realidad más concreta. Sin embargo, ocurre que la conservación de este proyecto primordial de la metafísica implica, al mismo tiempo, el vérsela con aquel concepto que más claramente lo ha alumbrado, el de la entidad.

Platón intentó organizar el ámbito del ser que establece el Ente en vista del concepto de «Idea», para devolver al ser, dentro de su unidad, una cierta multiplicidad, esto es, mayor abertura al ser concreto. El ser ideal sigue otorgando la entidad a todas las cosas, pero añadiéndole, además de la idéntica e imperecedera existencia, un orden, una imagen del cosmos, mediante los conceptos de comunidad y de participación. El ser ideal, que se expresa en las ideas, en definitiva, es otra analogía de atribución ( $\pi \rho \rho_{\varsigma} \varsigma$ ह̌v) de la realidad que sucede a la entidad de Parménides, que permite que lo que se atribuye no sea solo la existencia en sí, sino un orden para la totalidad. El paso sucesivo es el de Aristóteles: su concepto de ov̉ $\sigma i ́ \alpha$ es la categoría universal para comprender el ser, pero en cuanto ser concreto, el ser que se da primariamente a los sentidos.

La ov̉oía no atribuye al ser concreto la sola existencia, ni organiza esa existencia en "otro mundo" para alcanzar una unidad ideal dentro de la multiplicidad concreta. Aristóteles pretende que la oủ $\sigma i ́ \alpha$ sea la forma universal para todas las cosas a partir de la intuición que tenemos de ellas. Acabamos de señalar, sin embargo, con la ayuda de Ortega, que la intuición para Aristóteles es, en el fondo, intuición de la entidad. Si pensamos en la غ̇ं $\alpha \gamma \omega \gamma \eta ́$, en efecto, en la inducción mediante la cual Aristóteles busca alcanzar una intuición de los principios evidentes del silogismo, se in-duce, es decir, nos ad-entramos con ella en un reconocimiento o comprobación de las entidades universales que intuimos en los casos particulares (sean éstos individuos o especies referentes a su género). La idea clásica de la verdad como adecuación del pensamiento a la cosa es, en realidad, desde sus comienzos, adecuación del pensamiento a la entidad de la cosa, a los géneros y especies de los individuos, que son los límites de su nueva entidad. Pero no es otra que ésta, al final y al cabo, en mi opinión, la razón que obliga al Estagirita a dar el paso teórico de la sustancia primera a la sustancia segunda; se trata de un paso indebido para la percepción sensible, que no puede prescindir del movimiento y que, sin embargo, se adecua a otro modo de pensar, el de la entidad. El resultado es que lo primero a la experiencia para Aristóteles, es lo más difícilmente cognoscible para la razón, que es la razón de la identidad, de la no contradicción, de la entidad de las formas en la definición y en el silogismo. 
A raíz de este modo de pensar, Ov̉øía, desde entonces, es un engendro imposible, un compromiso entre dos modos de pensar irreconciliables, que pone definitivamente en evidencia la insuficiencia del concepto griego de razón, porque establece que el patrimonio íntegro del ser, su imagen íntegra y concreta que encontramos en nuestra alma, no es del todo cognoscible. El ser concreto, que es lo más real para los sentidos es, en el fondo, inalcanzable al discurso, porque la palabra siempre universaliza. La apertura a la sensibilidad que propugna Aristóteles esconde el racionalismo de Parménides, si bien enriquecido por la lógica platónica, perfeccionado mediante la definición, los predicables, el silogismo, etc.

Podemos concluir, pues, inspirados en la interpretación orteguiana del ente, que cuando Aristóteles afirma que el problema de la entidad no es otro que el de la sustancia ${ }^{7}$, hace algo que nadie se había atrevido a hacer anteriormente. Platón, sin duda, había intentado que la realidad concreta cupiese en el molde del Ente, que participara de algún modo de él, pero tal participación, siempre imperfecta, le había sugerido una saludable separación -por metafórica o pedagógica que se la quiera considerar- entre planos tan diferentes. Afirmar, sin embargo, como hace el Estagirita, que lo concreto es esencialmente lo abstracto, que la sustancia es la entidad, significa sugerir constantemente a la posteridad filosófica que la sustancia individual tiene que ser, de algún modo, un universal. De ahí, por supuesto, la inevitable homonimia del término ov̉ $\sigma i ́ \alpha$, su ser «sustancia primera» y «sustancia segunda», su ser forma, materia y compuesto de ambas, y su ser muchísimas otras cosas que buscan la improbable entidad de lo concreto. Es éste el dramático derrotero de muchos siglos sucesivos de pensamiento filosófico, y es éste, dicho sea de paso, en mi opinión, el origen real de la "eterna" polémica de los universales 8 .

Ahora bien, Ortega nos descubre un importante supuesto que lleva al descubrimiento de la entidad; de este supuesto hemos empezado a vislumbrar el génesis de algunas importantes dificultades, por no decir contradicciones, del pensamiento aristotélico, e, incluso, de la tradición sucesiva. Hemos intuido, por ejemplo, que la gran cuestión de los universales está presente, con toda su problemática, en el concepto aristotélico de ov̉øía. En el momento en que la razón griega es un decir con palabras el ser de las cosas que intuimos en el alma, se pone el problema de la adecuación de lo que intuimos, que siempre es concreto, con la universalidad del concepto que se expresa con las palabras. Todo esto es, sin embargo, solo un atisbo de todo lo que podemos comprender si instalamos nuestra mirada en la idea de la realidad que alumbra la filosofía orteguiana, que entiende que la vida es la realidad

\footnotetext{
${ }^{7}$ Cf. Metafisica VII 1, 1028a28ss y 1028b2ss.

${ }^{8}$ Confróntese esta interpretación con la que sugiere, por ejemplo, A. de Libera en su La querelle des Universaux. De Platon à la fin du Moyen Age, Éditions du Seuil, Paris, 1996. El compromiso imposible entre la entidad y la realidad concreta en el concepto de sustancia es, desde mi punto de vista, la verdadera incohérence de l'aristotélisme, su ambiguité destinale, para parafrasear el título de un apartado del libro citado (cf. p. 29).
} 
radical y que la persona, como descubre sobre todo Julián Marías, es su realización programática. En las páginas que siguen, intentaré mostrar que si la vida personal es la misma realidad haciéndose, el pensamiento griego ha ejercido una primera reducción conceptual de ella, ante todo, cuando ha asumido que la realidad verdadera es "el ser" en cuanto existencia de la púøıc. En segundo lugar, el ser "físico", desde Parménides, ha sido interpretado desde la pura lógica de la entidad. Cada ser de la naturaleza es Ente, que es lo existente en sí, independientemente de su movimiento, incluso del movimiento del intelecto que lo piensa. Una vez que la circunstancia de la vida se ha "naturalizado", que las cosas han perdido la evidencia, incluso etimológica, de su existencia pragmática $(\pi \rho \alpha ́ \gamma \mu \alpha \tau \alpha)$, convirtiéndose en entes, el gran problema de la filosofía, el mismo que constituye el ideal de su verdad, va a ser el de adecuar el mundo y el pensamiento a la entidad, es decir, darle al mundo la forma lógica de las esencias inmutables y eternas. Mostraremos detenidamente, entonces, que la función que tiene la palabra desde la razón vital, es radicalmente distinta respecto de la que le otorga la razón griega, de suerte que su uso, de cara a la comprensión de la razón vital, representa un tercer límite interpretativo o "reducción", como también la he llamado. Finalmente, como se ha anticipado, insistiremos en mostrar como el concepto de sustancia es el resultado de estas tres reducciones de la realidad radical que es la vida personal, y añade a ellas otra, decisiva, que acomete cuando pretende interpretar la realidad concreta de este mundo, la que se da primariamente a los sentidos, en vista de la forma sustancial, introduciendo la insoluble cuestión de su principio de individuación, particularmente problemático cuando la ov̉oía se intenta aplicar a la sustancia personal.

Siendo fieles al concepto de razón vital de Ortega, que es razón histórica, podemos entender que llevar a cabo una hermenéutica filosófica significa mirar, desde una idea de la totalidad (una metafísica), otra idea que, desde aquella, nos aparece como parcial, filosóficamente insuficiente, pero funcional al papel que ha desempeñado en un cierto momento histórico, por haber introducido a verdades sucesivas, comprensivas de ella, que la han reabsorbido de forma creadora' ${ }^{9}$. No es otra que ésta, en el fondo, la tarea con la que los auténticos discípulos han homenajeado a sus maestros en la historia de la filosofía, y no sólo en ella, si por verdad entendemos no sólo la verdad filosófica. La posibilidad de que una verdad sea una perspectiva que pueda intensificarse a través de su integración con otras perspectivas, en vista de una concepción unitaria de una verdad última que nos espera (si no en éste,

\footnotetext{
${ }_{9}$ Una posibilidad, esta de la reabsorción creadora, nunca garantizada por la mera sucesión temporal, sino por la verdad de un pensamiento. Como explica Ortega, puede haber innovaciones o arcaísmos, períodos de crecimiento y de decadencias.
} 
en "otro" mundo al que esta tarea misma nos pueda acercar), es la posibilidad intrínseca de quien vive la vida humana, que consiste en «reabsorción» de la propia circunstancia ${ }^{10}$.

He señalado que desde la razón vital, la realidad es nuestra misma vida haciéndose, con lo cual la estructura esencial de la vida en general corresponde a la estructura de la realidad. La famosa fórmula de Ortega, de 1914, es el enunciado analítico de su teoría de la vida: «Yo soy yo y mi circunstancia, y si no la salvo a ella no me salvo yo». Esta fórmula pertenece a lo que podemos llamar una «lógica formal» de la vida, que describe los elementos necesarios y, por ello, universales de toda vida. Julián Marías, discípulo de Ortega, ha contribuido de forma decisiva para que el nivel filosófico implícito en esta fórmula alcanzara su plenitud. Su Metafísica de la Persona es un ejemplo de reabsorción de la circunstancia, de integración de las perspectivas, de conservación y superación de la obra del maestro. Una de las innovaciones decisivas de Julián Marías, es el descubrimiento de la «estructura empírica» de la vida humana ${ }^{11}$. Sus elementos no forman una estructura universal, porque constituyen la estructura de la alteridad vigente entre los individuos en la sociedad. En su conjunto, como veremos, es necesaria porque es necesario que, en mi circunstancia, yo reciba una imagen vigente del hombre en general para que mis actos individuales tengan un sentido humano, sin embargo, su contenido concreto, esa misma imagen del hombre, cambia en la historia. Explicaré con más detalle, naturalmente, el sentido de esta teoría, sin pararme, sin embargo, por la economía de este escrito, en sus evidentes analogías con otras teorías, sobre todo fenomenológicas ${ }^{12}$. El propósito, en este caso, es mostrar desde la razón vital el tipo griego de razón histórica que ha justificado la formulación del ser y del ente como principios metafísicos de comprensión de la realidad, que han introducido al concepto de verdad como adecuación, que han determinado una función concreta de la palabra dentro de su lógica, y que, finalmente, han llevado a la idea aristotélica de ov̉oía.

Creo que la mayor fertilidad del pensamiento de Ortega invita a comprender a la persona, que es el primer «yo» de su famosa fórmula, como un proyecto (el segundo «уо») de comprensión personal, es decir, una constante e inagotable pretensión de saber quién soy. Esto significa que «circunstancia» es la totalidad de todo lo que encuentro y puedo encontrar (ő $\lambda \mathrm{ov}$ ) para encontrarme a mí mismo, para saber quién

\footnotetext{
${ }^{10}$ Como es sabido, Ortega, inicialmente, se refiere a la razón histórica de la verdad, a la posibilidad de integrar las perspectivas para componer una verdad superior, con el término de «perspectivismo».

${ }^{11}$ Cf. MARÍAS, J., Antropología Metafísica, Alianza Editorial, Madrid, 1970. Cf. también el capítulo IX de mi Libertad y Método, FUE, Madrid, 2005.

${ }_{12}$ Me refiero, sobre todo, a la distinción que hace Husserl entre lógica formal, expresiva de una ontología formal, y una lógica material, que da razón de las ontologías regionales. Remito, para un análisis de ello, a mis artículos «Meditaciones filosóficas I» y «Meditaciones filosóficas II», respectivamente en $\mathrm{n}^{\mathrm{o}}$ 134, Octubre-enero 2004-2005, pp. 73-78, y n 136 , marzo-junio 2005, pp. 77-86 de Cuenta y Razón.
} 
soy. Yo soy mi cuerpo, mi psique, mis neuronas, porque me encuentro con todo ello, al igual que me encuentro con la historia que me ha precedido, con mis padres, con el lugar en que he nacido, con el universo entero; todo ello me individúa. Yo, mi realidad plena, entonces, soy un proyecto de comprensión personal circunstancial.

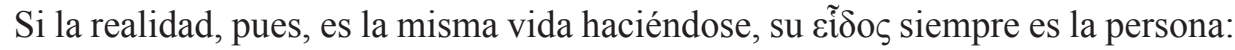
quien soy; y yo soy, quiera o no, consciente o inconscientemente, quien se elige con todo lo que me encuentro en un presente "transitivo", es decir, en un momento ahora que se hace futuro desde el pasado de quién he sido, y pasado desde el futuro de quién voy a ser. Desde esta perspectiva filosófica, en definitiva, como he sos-

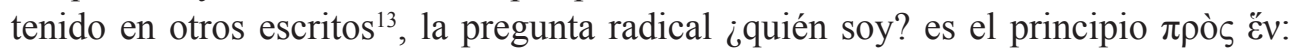
el principio en vista del cual podemos entender el sentido de toda la realidad. La totalidad (ö $\lambda \mathrm{ov}$ ) de la realidad se comprende, desde esta «analogía de atribución», como término y función de mi vocación.

Nada más esbozar estas ideas de una metafísica de la persona según la razón vital, podemos intuir, de inmediato, que la idea del ser que brota en Grecia, y que pretende comprender la totalidad de la realidad, solo alcanza una porción de ella.

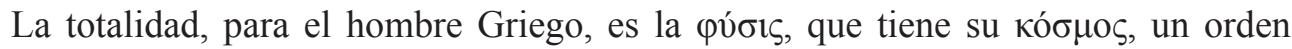
imperecedero, en sí, al que yo también participo en cuanto sustancia natural que tiene $\lambda$ ó $\gamma \circ \varsigma$, que es una facultad privativa del ser humano que le permite penetrar ese orden y aspirar a vivir según sus dictados. Esta observación, en primer lugar, nos desvela que la filosofía, así como aparece en Grecia, sin duda descubre una dimensión fundamental de la vocación personal, porque es búsqueda del sentido de la totalidad, de todo lo que nos encontramos; sin embargo, al no entender que esta vocación brota de la estructura de la vida humana, y que su sentido es esencialmente personal, se ejercen sobre la realidad y sobre el verdadero papel de la filosofía toda una serie de reducciones u ocultamientos. La primera, como hemos señalado, es confundir la vida con la púøıs, que es la creencia del pensamiento y de la cultura griega antigua, en general, de la realidad misma. La segunda, es la interpretación del ser de la naturaleza como entidad.

Hay que tener en cuenta, en principio, que al considerar que la pv́øıs es la to-

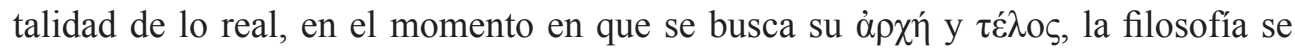
transforma en una "física" hecha con método filosófico, en una peculiar filosofía de "la naturaleza". Su vocación, en otras palabras, es filosófica, la de buscar el principio y el fin de la totalidad, pero esa totalidad no es la totalidad de lo real. Ello explica que, en el momento en que la matemática se aplica a la pv́бıs, broten de

13 "El idealismo dejará de ser de cosas, e incluso dejará de ser idealismo, sólo en el momento en que la filosofía se instale en la auténtica reflexión: aquella interpretación intrínseca en que consiste la vida humana y que obliga en todo momento a quien vive a comprenderse a sí mismo en su circunstancia, a saber circunstancialmente quién es. Lo cual implica que toda vivencia sea una idea concreta de persona, un esquema, figura o imagen concreta de la persona que en todo momento pretendo ser." DE Nigris F., "Meditaciones filosóficas (II)", p. 80. 
la filosofía, poco a poco, todas las ciencias. La naturaleza, en efecto, encuentra en la matemática su método de estudio más adecuado. La filosofía, entonces, conservará su pretensión pero perderá paulatinamente, con el avance de las ciencias, su campo de aplicación, de ahí que tendrá finalmente que replantearse su fundamento metafísico, preguntarse por lo que verdaderamente existe, por la efectiva capacidad de comprensión del ser. Es este, al fin y al cabo, el sentido de la modernidad filosófica, que con el cogito ergo sum de Descartes, pone en duda que el ser tenga primariamente una existencia natural, la que nos presentan las cosas en sí. El ser será, ante todo, el de la conciencia que nos presenta las cosas. La comunicación de las sustancias, de la res cogitans y de la res extensa, es decir, la cuestión del método, será, hasta prácticamente el siglo XX, la cuestión de la filosofía, que estará siempre amenazada por el peligro del solipsismo. Llamo «solipsismo» toda doctrina que muestra la incapacidad de la conciencia o de los actos del hombre de salir de sí mismos y tener una validez objetiva, podríamos decir, mundana.

Ahora bien, si bien los latinos nos han transmitido que el objeto de nuestro pensamiento es la cosa, la res, bajo este concepto, en realidad, solo se esconde un cierto tipo de cosa en sí, la entidad. La entidad ha hecho que las cosas perdiesen el matiz originario que conservaba su etimología $(\pi \rho \alpha ́ \gamma \mu \alpha \tau \alpha)$, ocultando, para la historia de la filosofía sucesiva, la función vital, absolutamente real, que tienen para la persona, esto es, para quién se descubre con ellas como parte de su circunstancia. Lo que quiero decir con ello es que la esencia de la verdad, entonces, para utilizar el título de un célebre libro ${ }^{14}$, no es la adecuación del pensamiento a la entidad del mundo, ni siquiera, como interpreta Heidegger, la vivencia de su desvelamiento $(\dot{\alpha} \lambda \dot{\theta} \theta \varepsilon 1 \alpha)$, a menos que se entienda como el desvelamiento inagotable de quien vive $\mathrm{e}^{15}$, es decir, a menos que se comprenda que la verdad es un encuentro revelador de quien soy con mi circunstancia, y que las cosas solo son una porción de ésta.

Aristóteles vislumbra este movimiento inagotable y personal de la vida humana, configurador de la verdad como personal encuentro circunstancial, cuando explora la forma del ser humano. Ve evidente que las facultades más nobles del alma, desde la sensible hasta, sobre todo, la racional, tienen un tipo de movimiento que es

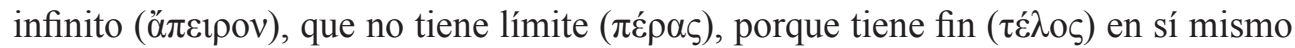

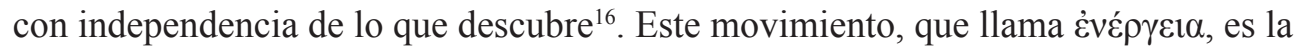

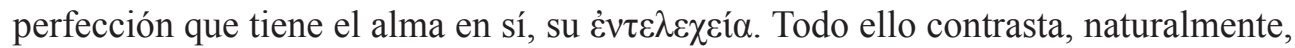
con el hecho de que el objeto de la verdad es el desvelamiento de la entidad, de las formas en sí de las sustancias, que tienen que ser acabadas, finitas, determinables

\footnotetext{
${ }^{14}$ Cf. Heidegger, M., Vom Wesen der Wahrheit.

${ }^{15}$ Cf. para la diferencia esencial de método entre Heidegger y la razón vital, mi artículo La razón vital de Ortega y Gasset y la analítica existencial de Heidegger, en Ideas y Valores, vol. 61, n. 148.

${ }^{16}$ Cf. Metafísica, IX VI. 1048b, 1050a 17 18ss; Acerca del Alma, 417a 16. Cf. Ortega y gasset, J., Prólogo a la "Historia de la filosofía de Émile Bréhier", en O.C. VI, pp. 409-415 y mi El origen griego de la intencionalidad medieval, "Paideia" nº 80, sept.-dic. 2007, pp. 335-351.
} 
una vez para siempre con el concepto que expresa la palabra. Esta contradicción se explica si entendemos que Aristóteles descubre el movimiento intrínseco de la vida a la vez que confunde su objeto, que no es la entidad en sí, sino el constante desvelamiento de quien soy con todo lo que me encuentro, mi circunstancia, que implica cosas, personas, e, incluso, si hace falta, entidades, por el estado del pensamiento en un cierto momento histórico.

Una de las mayores innovaciones de la razón vital, que se va explicitando en Ortega a lo largo de su doctrina como consecuencia inevitable de sus supuestos, y que se intensifica en claridad y sentido en la filosofía de la persona de Julián Marías -y que, permítaseme la apreciación, muestra ser tan poco atendida cuando se intenta comparar la razón vital con la estructura intencional de la conciencia fenomenológica-, es el cambio radical que asumen en ella los tradicionales conceptos filosóficos de "sujeto" y "objeto". He definido más arriba a la persona, que he identificado con el primer "yo" de la fórmula de Ortega, como un "proyecto personal de comprensión circunstancial". En efecto, la persona es el sujeto y el objeto de la vida humana, pues quien vive tiene que dar respuesta a quién es proyectando una figura concreta

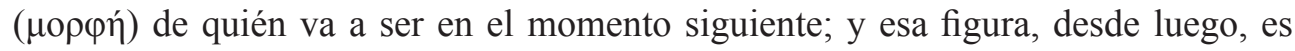
corporal, es física, química, psicológica..., pero no es esencialmente ninguna de estas dimensiones, que son las que abrazan los conocimientos científicos o, entendemos ahora, la filosofía cuando se entiende como ỏ $\rho \chi \eta ́ ~$ y $\tau \hat{\imath} \lambda o \varsigma$ de la naturaleza. Ser persona consiste en quehacer, en proyecto, en verdadera «ciencia buscada» ${ }^{17}$, porque es el argumento inagotable que se manifiesta en la vida humana, mostrando su capacidad de comprensión o amor. Una cosa es, pues, que los conocimientos científicos no caigan en demostraciones circulares o regresos al infinito, por no tener un principio de demostración, mas otra cosa es que se olvide, por esa necesidad científica, de la infinitud del movimiento esencial de la vida humana, cuyo principio es inagotable, la persona, y cuya realización, entendemos ahora, posibilita el mismo conocimiento científico que se ocupa de ciertas dimensiones suyas, que responden a su estructura empírica.

He mencionado que Heidegger afirma que la vivencia griega de la verdad ha solido verse desde su dimensión de «adecuación», la del ser del pensamiento al ser de las cosas, sin percatarse de que el hecho primordial de la verdad está expresado por su misma etimología, la del descubrimiento $(\dot{\alpha} \lambda \eta \dot{\theta} \theta \varepsilon 1 \alpha)$, del poner a la luz lo que está oculto. Ahora bien, acabamos de ver que lo que se desvela es el ser, y que el ser es el ser de la naturaleza, con lo cual su sentido pierde, como he dicho, consistencia filosófica. Pero estamos viendo con claridad que hay otra cuestión

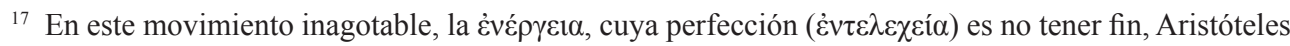

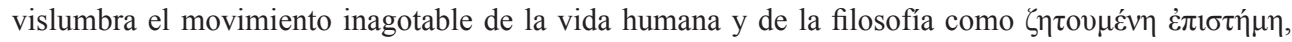
como ciencia radical de la vida. Es ésta, en mi opinión, la raíz última de esta definición tan famosa como discutida.
} 
decisiva: que el concepto de $\dot{\alpha} \lambda \hat{\theta} \theta \varepsilon ı \alpha$, si se toma en su sentido íntegro, implica un movimiento, el de quien descubre, y no solo su resultado, aquello que se descubre. Si se separa la cosa del proyecto que la descubre, pierde sentido su verdad. La consecuencia de esta "mutilación" es que la realidad es determinada por las formas de las cosas, y no por la verdad de la capacidad del intelecto de adecuarse a ellas. Esto, que le pasa al pensamiento griego, es lo que más rigurosamente tenemos que llamar «determinismo».

El peligro de todo determinismo es que, al imponérsele la realidad desde fuera al hombre, éste pierde su capacidad de hacerse real desde dentro, desde sí mismo; pierde, en definitiva, algo tan esencialmente humano como la libertad. Cuando no se reconoce que el objeto de la vida humana es una idea de quién soy, que lanzo hacia delante -ob-yectum- desde un presente transitivo, los objetos (cosas, entes, ideas, en definitiva) funcionan como lo absoluto al que los conceptos aspiran, por lo cual, el movimiento de la razón, como alumbra la razón griega, es libre, en el fondo, solo para buscar el camino que le lleve a un único destino, al ser esencial de todas las cosas, a su entidad. Y una prueba fehaciente de la fuerza con que las cosas han determinado nuestro pensamiento desde la más antigua tradición helénica, la tenemos en la revolución copernicana de Kant. La gran innovación del filósofo de Könisberg ha sido afirmar que es el sujeto, mediante la estructura a priori de su sensibilidad y de su intelecto, el que determina el ser de las cosas que nos aparecen; sin embargo, cuando vamos a ver las categorías a priori del intelecto nos reencontramos, aproximadamente, con las categorías de la sustancia aristotélica, es decir, con formas y relaciones aptas para pensar cosas. Su principio pretende ser revolucionario, pero su aplicación se limita a las ciencias, en las que el hombre solo observa cosas. La investigación de lo más propio de la vida humana en Kant (libertad, moralidad, conciencia, Dios...) es tema de la razón práctica, que, sin embargo, no llega a ser la razón vital, en la que se descubre que siempre, todo "objeto", del pensamiento o de la naturaleza, tiene una existencia pragmática al descubrimiento del verdadero objeto de la vida humana, ese quién concreto que proyecto desde el presente de mi vida y al que intento adecuar mi circunstancia, para alcanzarlo, en un presente sucesivo.

Manteniendo la estructura sistemática de nuestra narración, se nos presenta, ahora, como consecuencia de lo dicho, uno de los problemas más grandes que levanta la doctrina aristotélica desde el ser sustancial de la púoıs: la interpretación de la persona como oủoía y su principio de individuación. Pretendo, en el próximo apartado, si bien esquemáticamente, estudiar el origen del problema, que radica en la misma dificultad que tiene la sustancia aristotélica para individuarse. Intentaré mostrar, entonces, que lo que llamo el principio del movimiento de la vida humana, su auténtico principio $\pi \rho o_{\varsigma} \check{\varepsilon} v$, que es la pregunta radical ¿quién soy?, es, también, el principio de individuación y de la alteridad que constituye su objeto, la persona. 
A lo largo de los siglos se han dado básicamente dos interpretaciones. La tradicional, apoyada por Santo Tomás, por ejemplo, ha entendido que la sustancia es una

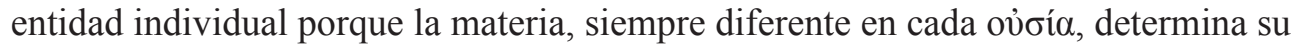
forma universal. Y, por otra parte, aquella que a lo largo del siglo XX -sobre todo en su segunda mitad- ha tenido cada vez más seguidores, y entiende que la individualidad de una sustancia concreta es la de su forma ${ }^{18}$. Las hacceitas de Escoto, quizá, pueden considerarse un ilustre antecedente a esta doctrina.

Vamos a suponer, en primer lugar, que es la forma, en cuanto entidad individual, lo que determina todo el ser de la sustancia, incluso su potencialidad. Resultaría, entonces, como parece a veces admitir Aristóteles, que la materia es un correlato funcional al estado de desarrollo esencial de la sustancia, esto es, al estado actual de su última perfección o entelequia. Sin embargo, por lo pronto, si consideramos que la definición se da sobre formas ingeneradas y eternas, y que el ser de este mundo está siempre en movimiento, resulta que deberíamos pensar en un criterio para establecer que lo que definimos sea realmente el estado final y acabado del desarrollo potencial de la sustancia, el que manifiesta su forma plenamente actuada. Podríamos pensar, entonces, en comparar dicha sustancia con el estado de desarrollo de otras pertenecientes a la misma especie; así, sin embargo, las dificultades se ramificarían. En primer lugar, porque si las sustancias tienen una forma últimamente individual, ¿cómo podríamos hablar de sustancias pertenecientes a una misma especie?, ¿con qué criterio elegiríamos un punto de referencia específico para cada una que fuera real y no puramente conceptual, resultado de la costumbre, como diría Hume? Y con estas dificultades nos introducimos directamente en otra que ya conocemos: ¿si la forma es individual, podemos realmente reflejar su ser mediante conceptos y definiciones? Aristóteles lo niega, porque necesitaríamos de un nombre único para cada especie ${ }^{19}$, lo cual es imposible, y, si lo fuera, no solo no daría lugar a una ciencia, sino ni siquiera a un lenguaje, porque todo lenguaje para comunicar necesita generalizar.

Pero el problema de esta concepción no termina aquí; es aún más grave de lo que parece. Tanto la filosofía platónica como la aristotélica, cada una a su manera, encuentran en el carácter generador de la esencia el orden permanente e intrínseco al movimiento de la naturaleza. El ser es lo que es ( $\tau$ ò $\tau$ í $̇ ं \sigma \tau)$ por ser lo que era

\footnotetext{
${ }_{18}$ Algún ejemplo de eminentes trabajos que han apoyado esta concepción: Frede, M. y Patzig, G., Metaphysik Z, Text, Übersetzung und Kommentar; Bd.I-II, Beck, Munchen, 1988; E. Hartman, Substance, Body and Soul. Aristotelian Investigations, Princeton, 1977; C. WIтT, Hylomorphism in Aristotle, Apeiron, 22, 1989, pp. 141-158. El mismo Giovanni Reale reivindica esta interpretación diciendo que fue por él apoyada desde su obra de 1961: Il concetto di "filosofia prima" e l'unità della metafisica di Aristotele, Milano, Vita e pensiero; cf. esta afirmación en su Guida alla lettura della "METAFISICA" di Aristotele, Roma-Bari, Laterza, 1997, p. 146.

${ }_{19}$ Metafísica, V 29, 1024b 33ss.
} 
( $\tau$ ò $\tau$ í $\tilde{\eta} v$ Eĩval); una sustancia genera otra de su propia especie, lo que establece un orden inmanente a la naturaleza ${ }^{20}$ y determina una concepción del azar y de la suerte, respectivamente, como fallo y resultado azarosamente positivos que contribuyen a ese mismo orden ${ }^{21}$. En la concepción ontológica de Aristóteles, la especie, a pesar de ser menos abstracta que el género, porque conceptualmente es más cercana a la realidad concreta, todavía permite una comunidad genérica entre sustancias primeras, ordenando su generación y conocimiento. Admitir, sin embargo, que cada sustancia tenga una especie única sería automáticamente hacer de la mutación genética el principio ordenador de la naturaleza. De una especie se generaría otra últimamente distinta, con lo cual, ¿cómo podría ser una especie el principio generador de otra? ¿Qué proporciones o razones podrían explicar el paso de una a otra? Lo más seguro, en definitiva, considerando el proyecto epistemológico de Aristóteles, es que de esencias individuales se derive la imposibilidad del conocimiento y la infinita complicación del orden inmanente de la naturaleza, hasta

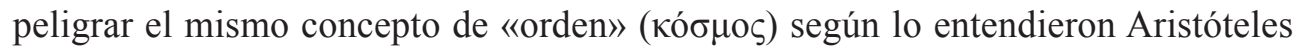
y Platón.

En cuanto a una concepción clásica de la sustancia, si el principio de individuación de cada cosa no depende de la forma, que es universal en cada especie, sino de la materia, entonces resulta que lo más propio y posiblemente valioso de cada realidad, incluso su belleza individual, dependería de factores contingentes. Esto, que resulta ya difícil aceptar para las cosas o los animales, llega a ser inaceptable para la persona. Su individualidad sería algo inesencial y caduco, y, por supuesto, seguiría siendo indefinible. Esta cuestión nos muestra con evidencia la raíz aristotélica del problema medieval de la creación, salvación y conservación del alma individual en el pensamiento medieval cristiano.

Vemos, además, que desde el punto de vista epistemológico seguiría en pie el mismo problema: la definición, siendo del universal, no permitiría comprender lo peculiar de cada sustancia. Es verdad que en este caso significaría no comprender lo más caduco de ella, sin embargo, remitiéndome a lo que acabo de decir, lo más caduco sería en muchos casos lo más decisivo, por ejemplo, la personalidad única

\footnotetext{
${ }^{20}$ Cf. Ibid. VII 7, 1032a 11-15; VII 8, 1033b 20-20 y 1034a 1-7; XII 4, 1070a 25-30.

${ }^{21}$ Desde el punto de vista del arte, el orden sería el fin de la obra, lo cual haría del azar lo imprevisto (no perseguido intencionalmente) que puede incurrir en la consecución de ese fin, y la fortuna (suerte) como el carácter positivo de lo que sin querer se hace -o simplemente ocurre- de cara a ese mismo

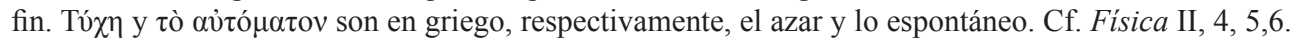
El primer término se refiere al azar entendido como «caso» y, a la vez, como «suerte» o «fortuna». Aristóteles habla de la necesidad del accidente en su obra biológica, cuando admite la posibilidad de obras monstruosas en la naturaleza. Éstas en principio contradicen sus leyes, pero en el fondo, tomada la naturaleza en su conjunto, son accidentes necesarios para el desarrollo natural de todas las cosas. Lo contingente, lo accidental, en este sentido, es esencial al mundo. Cf. también Sobre la generación de los animales IV 3, 767b 11-16.
} 
de cada ser humano ${ }^{22}$. Finalmente, a diferencia de la concepción anterior, considerar la materia como principio de individuación haría muy problemática la noción aristotélica de «materia primera» o amorfa, que es la realidad más cercana a la nada y más alejada de dios. Nadie, en efecto, tiene conocimiento de la materia amorfa excepto como "algo" que podemos solo imaginar por estar exento de toda determinación. La materia siempre se nos presenta informada, de suerte que es ininteligible si no es en vista de una forma: el árbol es materia para una pata, que es materia para una mesa, que es materia para el fuego...

Cuando Aristóteles dice que la materia también es ovoía $\alpha^{23}$, parece más verosímil esta concepción tradicional en que la materia es una realidad sustantiva que individúa la forma; sin embargo, cuando parece verse en la materia el halo de virtualidad de la sustancia, ligado al estado de actualidad de la forma, se hace más verosímil identificar completamente la sustancia con su forma. Ambas interpretaciones, sin embargo, como vemos, siempre acaban por resultar contradictorias. La aporía, por un lado u otro, no se soluciona; y la razón de ello no es otra, en mi opinión, que la que está a la raíz del concepto mismo de sustancia: el compromiso imposible entre la entidad y la realidad concreta. Esta carga aporética se intensifica especialmente cuando desde el molde de la sustancia se pretende entender a la persona,

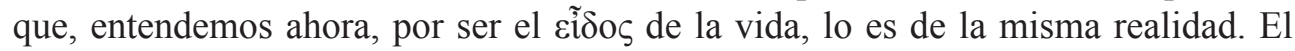
pensamiento cristiano, que tiene en el centro de su especulación este concepto, será el que encontrará más dificultades para definir tanto a la persona humana como a la Divina que se manifiesta en el Evangelio.

Es la visión del hombre como sustancia, como compuesto de alma y cuerpo, que no permite resolver el problema de la personalidad. La $\psi v \chi \eta ́$ remite a un conjunto de potencias o facultades que tiene el hombre por naturaleza. Podría pensarse que el alma tiene unas facultades universales y que es el cuerpo, distinto para cada persona, lo que las individúa, o, por otro lado, que cada alma tiene unas facultades o talentos absolutamente únicos, pero con ello no encontramos todavía el principio de su personalidad. La persona no se constituye por lo que tiene, por lo que es. Si queremos remontarnos a una visión materialista moderna, que, en mi opinión, ofrece dificultades muy parecidas a las del alma aristotélica, podemos afirmar que el ADN, en cuanto material genético del hombre, no es capaz de individuarlo hasta hacer de él alguien único, un quién concreto. Esto se nos muestra con evidencia en la clonación. En ella se reproduce un «qué», un patrimonio material, pero no quién es cada individuo, que tiene su intransferible circunstancia, resultado de sus proyectos. Se puede reproducir lo que es el hombre, su materia, pero no quién es.

\footnotetext{
${ }_{22}$ Dice Aristóteles que «Si tratamos de definir alguna cosa individual, siempre se puede refutar, porque no es posible definirla» Ibid. VII 15, 1040a 6-7; sin embargo, a diferencia de lo que hace a continuación con las ideas platónicas, que considera realidades separadas e individuales (Cf. 1040a 8-10), no considera esta dificultad sistemáticamente en relación con su doctrina de la sustancia.

${ }^{23}$ Cf. Metafisica, VIII 2, 1042a 25ss.
} 
Y esto nos devuelve, repentinamente, a la importancia del concepto de «creación», tan propio del cristianismo y, como vemos, de la estructura de la vida humana. Cada persona, decía Julián Marías, es «una radical innovación de realidad», el ejemplo vivo de la actividad personal creadora de Dios para con los hombres, y que éstos, hechos a imagen y semejanza suya, celebran en la concepción de cada hijo como alumbramiento de una realidad única, nunca antes existida.

Aristóteles distingue entre potencias congénitas y adquiridas del alma. Estas últimas no se activan automáticamente como el respirar o el crecer, que son potencias congénitas, sino mediante el deseo y la elección ${ }^{24}$. ¿Pero, cuál es el deseo último del hombre, el que puede últimamente hacerle feliz, hacia el cual deberían tender todas sus facultades? Es la vida, dice Aristóteles, que sabe vivir conforme a su naturaleza. La naturaleza del hombre, el principio de su movimiento, es el alma, y su facultad más noble es la racional. Sin embargo, ¿puede ser la coincidencia del pensamiento con el ser de las cosas el proyecto ético del hombre? ¿Puede ser el descubrimiento del orden del cosmos "físico" su proyecto felicitario? Si para el ser humano no se asume un principio de personalidad distinto del modo de obrar de la naturaleza, si encuentra una razón personal a su movimiento anímico, su cosificación, sea en forma de ente, de idea, o de sustancia, significa la pérdida de sentido de la vida personal.

La vida, en realidad, se mueve a partir de una radical cuestión personal, ¿quién soy?, y todos sus proyectos se configuran como sucesivas respuestas personales que acontecen en un presente que he definido «transitivo». La vida, en su estructura universal, es constante reabsorción de sí misma: en cada proyecto presente está implicada la vida entera a la vez que complicándose, es decir, su pasado transita hacia el futuro en una forma personal siempre nueva a todas las anteriores, pero incomprensible sin ellas. Esto quiere decir que mi vida, y la realidad por ser la misma vida haciéndose, es siempre «sistema»: cada uno de sus elementos implica la totalidad de lo vivido, y, la totalidad, cada uno de sus elementos. Es verdad, por otra parte, que si ésta es una apreciación que pertenece a la estructura analítica de la vida humana, que vale para cada vida, independientemente del contenido de sus proyectos, resulta que el conocimiento que tenemos de ella, y que se apoya en su misma sistematicidad, puede no ser sistemático. La incoherencia, la falta de sentido de nuestros proyectos es posible por la estructura sistemática que tiene, a priori, nuestra vida, que se hace siempre en vista de la misma cuestión. Como la vida analíticamente se hace de sentido, mis proyectos, de cara a ella, pueden concreta y aparentemente no tenerlo, pueden, en otras y más precisas palabras, ocultar su sentido personal ${ }^{25}$. Veremos, más adelante, que a partir de estas observaciones podremos renovar los conceptos de «materia» y de «forma» para una nueva concepción de la

${ }^{24}$ Cf. Ibid., IX 5, 1048a 10-12.

${ }^{25}$ La despersonalización de la vida humana como sentido primario de su inmoralidad. Cf. MARÍAs, J., Tratado de lo Mejor, Alianza, Madrid, 1995. 
persona en cuanto «sustancia». De momento, es fundamental subrayar el siguiente aspecto de cara al problema de la individuación personal. La respuesta irreductible de cada vida a la misma cuestión radical de su personalidad, permite que haya una unidad del proyecto del ser humano que postula sus diferencias individuales, las personas, y hace que éstas, a diferencia de los animales, tengan historia. En otras palabras, siendo la cuestión personal de la humanidad una y la misma para todas las personas, cada una se diferencia por cómo se comprende en su circunstancia, por cómo se individúa en ella, por su historia o biografía.

Ahora bien, el sentido radical de «comprender», entonces, es el de abarcar todo lo que me encuentro por y para aprenderme o encontrarme a mí mismo ${ }^{26}$. Sin embargo, esto solo lo puedo hacer si en mi circunstancia me encuentro con otras personas que tienen mi mismo proyecto. Frente a una silla soy quien se sienta, frente a un cuchillo soy quien corta, pero solo frente a un tú, como expliqué, puedo ser plenamente «yo», porque se me manifiesta mi personalidad. Solo encontrándome en otra vida única, biográficamente insustituible, me descubro como yo mismo, en el ámbito único de posibilidades de personalización que es mi vida. El principio de individuación de la vida humana, entonces, es, a la vez, el principio de su alteridad, porque como intuía Unamuno con maravillosa expresión: «Una persona aislada deja de serlo. ¿A quién, en efecto, amaría? Y si no ama no es persona» ${ }^{27}$.

Vamos ahora abordar, a raíz del concepto de "compresión" que acabamos de esbozar, y del principio de individuación de la vida humana que propone, otra cuestión fundamental que la razón vital puede esclarecer acerca del pensamiento sustancialista aristotélico: la función de la palabra.

Hemos comprobado que las palabras no pueden dar razón del ser concreto, sea éste un compuesto de materia y forma, sea una especie individual. La ineptitud de la universalidad de los conceptos para la tarea que la razón griega les asigna, nos tiene que mostrar que la lengua, en realidad, tiene otra función dentro de la vida humana. Ante todo, la lengua no es una "lógica", en el sentido de un lenguaje construido intelectualmente, como la lógica aristotélica, por ejemplo, que busca definir la realidad a partir de una idea de ella, la naturaleza organizada en sustancias con formas genéricas y específicas. «Hombre», por ejemplo, a través de este sistema teórico, se define como «animal racional». Hay también lenguajes científicos que buscan definir ciertas porciones muy específicas de la realidad, cuyos signos, a menudo, no son siquiera palabras, como ocurre con las matemáticas. Los términos de la lengua,

\footnotetext{
${ }^{26}$ Cf., De Nigris, F., "La persona en su proyecto de comprensión" en Libertad y Método.

${ }^{27}$ Unamuno, M., Del sentimiento trágico de la vida, Editorial Plenitud, 1966, Madrid, cap. VIII, p.149.
} 
en general, no se suelen fijar intelectualmente, no son «constantes lógicas» que se definen con una cierta función significativa, sino que se forman y varían a través de la convivencia. Habrá que preguntarse, entonces: ¿cuál es la verdadera lógica de la lengua, aquella de donde brotan y se organizan sus conceptos?

Desde la razón vital se entiende que la lengua, también, tiene una lógica intrínseca que depende de la radical cuestión personal de la realidad: ¿quién soy?, de ahí que se define como el principal sistema de signos verbales que permite la comprensión personal del individuo en la convivencia. En la metafísica de la persona que brota de la razón vital, hay una auténtica superación de todo solipsismo, y la lengua, así interpretada, es una evidencia de ello. He mostrado que «yo soy yo y mi circunstancia» significa que cada prójimo con el que me encuentro, es

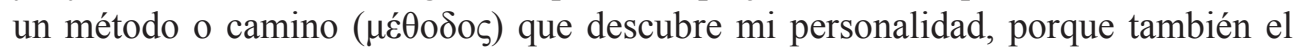
prójimo, al vivir una vida humana, tiene una vocación de descubrirse en vista de mi vida. Hay una esencial interpenetración reveladora de las personas sin la cual el concepto mismo de «persona» sería imposible. La lengua, pues, es un sistema de signos que permite la comprensión personal, es decir, la universalidad de sus conceptos es funcional para que cada individuo, dado un esquema «lógico común» (el conjunto de significados que encontramos en el diccionario y que son el resultado de la convivencia de un pueblo), lo utilice con una «función significativa» única en su vida ${ }^{28}$. No puede haber individualidad sin universalidad.

Julián Marías, en su genial libro Introducción a la filosofía ${ }^{29}$, distingue del concepto de un término su esquema lógico y su función significativa concreta. Un término tiene un esquema lógico que puede actualizarse con diferentes funciones significativas, dependiendo de la situación concreta en que se utiliza, esto es, según «el decir concreto de la vida» que le subyace. «Pintar», dice Marías, no significa lo mismo para el hombre del paleolítico de Altamira o para Velázquez, así como «racional» no significa lo mismo si se piensa en vista de Greta Garbo o de Santa Teresa, de Platón o de Aristóteles; sin embargo, hay un núcleo idéntico que permite que yo hable de «pintar» y de «racional» para todos estos y más casos. Ese núcleo, en la lengua, como acabo de mencionar, es el conjunto de significados que recoge su diccionario correspondiente. Con esta aclaración terminológica, podemos seguir con tres ulteriores importantes observaciones.

En primer lugar, se entiende la razón de algo tan cotidiano como la posibilidad del malentendido. El habla, por la universalidad de los esquemas lógicos de sus conceptos, implica siempre dos reducciones para con la vida: la del hablante que no puede expresar del todo lo que le pasa a sí mismo con palabras, y la del oyente

\footnotetext{
${ }^{28}$ MARÍAs, J., "El problema de la lógica", apartado 61 de Itroducción a la filosofía, Tomo II de Obras, Madrid, Revista de Occidente, 1992.

${ }^{29}$ Cf. MARÍAs, J., Introducción a la Filosofía, en tomo II de Obras, apartado 45 del cap. V y el 61 del VII.
} 
que interpreta las palabras oídas desde su vida, desde su ámbito de razón. Esto se verifica claramente cuando hay dos vidas que se habitan profundamente. Suele disminuir, en estos casos, la equivocidad de los términos. Por otra parte, el esquéma lógico tampoco es una «constante lógica». He señalado que la lengua no fija sus significados intelectualmente, sino a través de la convivencia. Eso quiere decir que sus contenidos tienen cierta permanencia que permite la comunicación, pero esa misma comunicación interindividual es el principio de su constante cambio. Podríamos decir, con términos fenomenológicos, que la implección significativa de un signo intuitivo (como la palabra, por ejemplo) va, mediante su uso vital e histórico, moldeando su esquema lógico, es decir, su función significativa. En tercer lugar, todo esto nos permite entender que la sistematicidad de la lengua radica en la sistematicidad de la vida personal. En el decir lingüístico una palabra se entiende en relación con el sistema concreto de su lengua; ésta, a su vez, desde el conjunto de vivencias del pueblo que la habla, y, como quienes la hablan siempre son individuos, de la vida concreta de cada uno como el ámbito en que radica su sentido último, como el ámbito en que sus esquemas lógicos se actualizan en funciones significativas concretas. Y, finalmente, en esta actualización entre individuo y lengua, entre la dimensión irreductible de la persona y su dimensión social, como acabo de decir, la lengua se va moldeando. En la «reabsorción de la circunstancia» cada vida reabsorbe también la lengua que es porción esencial de ella, hasta que sus cambios, que pueden tener inicialmente una variación en un pequeño grupo, incluso de amigos, pueden llegar a tenerla en una sociedad entera. Salta a la vista, desde esta perspectiva, la enorme función que tienen los medios de comunicación en difundir la vigencia de los conceptos y, en general, el uso de una lengua. Creo que sería interesantísimo ver hasta que punto la literatura, en ciertas épocas, ha podido tener esta esencial función, y lo que ha implicado su supuesta pérdida.

Por «sociedad», recordémoslo, Julián Marías entiende un grupo de personas que vive bajo un sistema de vigencias comunes ${ }^{30}$. Las vigencias son aquellas interpretaciones (ideas, creencias, opiniones, incluso sentires...) que perfilan una figura de «hombre» común a los individuos, en vista de la cual cada uno puede entender los propios actos y aquellos ajenos con un sentido «humano». Esas interpretaciones no solo se refieren a lo que es vigente en el mundo "interior" (ideas y creencias, por ejemplo, sobre la libertad, la amistad, la diversión, la existencia de Dios...), sino también en su dimensión física. "Creemos", es decir, damos por hecho en la vivencia de nuestra corporalidad humana, que tenemos dos brazos, dos piernas, dos ojos, que podemos correr a una cierta velocidad, que contamos con una cierta tecnología... Es la idea vigente del hombre en vista de la cual nos proyectamos, es la imagen del mundo en cuanto «nostridad», es, como la define Julián Marías, la estructura empírica de la vida humana. Esta estructura, dice Julián Marías, es así,

${ }^{30}$ Cf. La estructura social, Colección El Alcion, Madrid, Editorial Revista de Occidente, 1972, p. 39. 
pero podría ser de otra manera, de hecho cambian a veces sorprendentemente sus elementos. Y si imagináramos la posibilidad de vivir en «otro mundo», después de nuestra muerte, por ejemplo, podríamos suponer que conservaríamos nuestra estructura analítica, el proyecto personal de comprensión circunstancial que nos define como personas, pero, quizá, con otra psique, con otras formas de comunicación, con otra capacidad de comprendernos, en definitiva. La lengua, es, pues, otra dimensión empírica de «el hombre» que cada uno somos; es, como he explicado, la principal interpretación conceptual verbal del mundo.

Si ahora volvemos al tipo de razón griega, entendemos que la función que ésta ha otorgado a la palabra, ha encubierto su auténtica razón vital. Las primeras explicaciones del fenómeno humano del lenguaje han tenido que vérselas también con la creencia en la naturaleza. El hombre por naturaleza habla, pero, ¿son sus palabras y conceptos reflejos del orden de la pv́бıఢ, o, simplemente, responden a la "ley" humana (vónos) del acuerdo y de la costumbre? En el Crátilo y en el Sofista, Platón nos expone con tal fuerza retórica las dos posturas, que muchos intérpretes han dudado del punto de vista del mismo autor. Según los partidarios de una explicación natural, la etimología de las palabras tiene una intrínseca vinculación con las leyes de la naturaleza. El movimiento del $\lambda$ óyos del hombre sigue el ritmo del

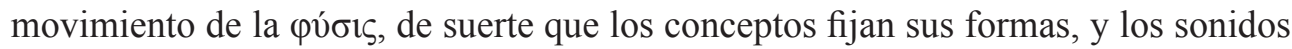
de las palabras, como ocurre con la onomatopeya, fijan el contacto sensible que tenemos con ella. El problema surge, sin embargo, cuando se consideran lenguas distintas, con formaciones etimológicas distintas, lo que pone en duda la unidad del orden natural o la capacidad humana de interpretarlo. Ésta y otras dificultades conducen, sobre todo a los sofistas, a una interpretación de los conceptos como esquemas funcionales a la variabilidad de lo real, que tienen en su origen el acuerdo humano y la costumbre. De una manera u otra, en realidad, no se entiende la lógica vital subyacente a la identidad y a la variabilidad de los esquemas lógicos de la lengua, ocultando su verdadera función para la comprensión individual en la convivencia. La lengua, para el proyecto racional griego, tiene que describir la entidad del ser concreto, una contradicción, ésta, que se manifiesta clara y definitivamente en el sistema de Aristóteles, en particular modo en la dicotomía entre $\pi \rho \omega ́ \tau \eta ~ o v \sigma i ́ \alpha$

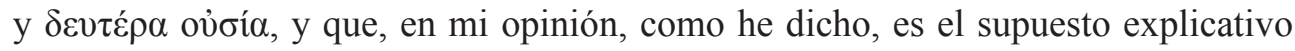
de la enorme cuestión medieval de los universales.

\section{IV}

A raíz de nuestra hermenéutica según la razón vital aplicada al concepto de ovoí $\alpha$, podemos perfilar las siguientes conclusiones. Ante todo, el concepto de «entidad» que recibe Aristóteles ya implica una triple reducción de la razón íntegra de la vida. Acabamos de ver que el proyecto de ésta no es un decir con palabras el ser de las cosas. La vida ni piensa solo con palabras, ni dice, en su sentido íntegro, solo 
lo que se puede conceptuar con ellas. Los conceptos que expresan las palabras son como islotes que nos orientan en el océano de nuestra vida, cuyas vivencias, si bien sistemáticamente entrelazadas, tienen distintos niveles de conciencia y de presencia conceptuada verbalmente para quien vive. Como he dicho anteriormente, una cosa es el contenido entero, sistemático de la vida, otra es la comprensión conceptual verbal que tenemos efectivamente de ella. En segundo lugar, he mostrado, desde el comienzo de este escrito, que «decir el ser» no es la razón esencial de la vida,

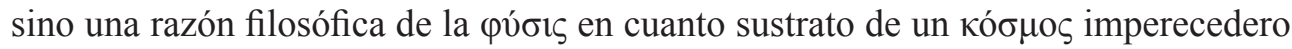
del que brotan y se justifican todos los seres. Finalmente, el concepto sucesivo de entidad con el que Parménides define el ser, es, a su vez, la reducción de ese ser "físico" a un conjunto de propiedades lógicas que lo definen. Cada ser es Ente, es lo existente en sí, independientemente de su movimiento, de todo lo que pueda acontecerle, incluso, paradójicamente, de que yo lo piense, porque, en efecto, si el pensamiento piensa otra cosa que el ser, se equivoca.

Si la palabra, pues, reduce el ámbito de la razón vital a lo que se dice verbalmente, y el ser a lo que se dice de la púøıs, la entidad, respecto de ese ser, se refiere solo a su existencia en sí, inmutable, idéntica a sí misma, imperecedera, supuestamente independiente de mi. A partir de ahí, durante casi toda la historia de la filosofía sucesiva, el problema del ser, en su forma de entidad, como ya dije, se hace «el» problema de la metafísica, olvidando a menudo la cuestión del sentido del concepto de entidad, de lo que implica y complica el «ente» para la comprensión de la vida humana. Ovoía, finalmente, en cuarto lugar, es el forzoso compromiso entre dos proyectos intelectuales distintos, porque declara que la entidad es, en el fondo, la realidad concreta, esto es, que dos formas distintas son la misma.

En su conjunto, estas cuatro "reducciones" son, en mi opinión, la distancia teórica que separa la filosofía aristotélica de la razón vital. La sustancia, en cuanto ente natural, no permite comprender la vocación personal de la vida humana, su principio de individuación, la función de la lengua, la universalidad de sus conceptos, la alteridad como parte esencial de su constitución individual. Esta distancia también ha separado el alma occidental, inevitablemente, de una comprensión teórica de otra de sus raíces históricas, el cristianismo. La lógica de la sustancia natural ha resultado especialmente desorientadora para la interpretación filosófica del mensaje evangélico, ha cristalizado en el pensamiento de la Iglesia la clásica definición de la persona como rationalis naturae individua substantia, por decirlo en términos de Boecio, con todo aquello que, estamos viendo, implica el concepto clásico de «ratio»y de «substantia».

Ahora bien, habíamos prometido, finalmente, buscar una reformulación del concepto de sustancia de suerte que nos fuera posible comprender con él a la persona, que es el proyecto radical de la vida humana. Para ello tenemos que pasar por un descubrimiento fundamental que hace Aristóteles, con el que contribuye de forma decisiva al futuro desvelamiento de la razón vital. Me refiero al gran descu- 
brimiento del movimiento inmanente de la realidad, que describe con el término

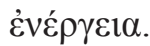

Por dónde se coja la doctrina del Estagirita nos encontramos reiteradamente con la idea de lo que es por sí y lo que es por otro. Es la relación que distingue esencias y accidentes, actos y movimientos, alma y cuerpo, artes poiéticas, prácticas y teóricas; o que funda en la autarquía toda clase de perfección ética, política y ontológica en general, y, finalmente, es la única clase de infinitud que admite con sentido: la que es propia de sustancias que cierran el círculo de su actividad sobre sí misma, lo que impide el «regreso al infinito» y permite una intensificación de su ser. Aristóteles descubre este movimiento, como he anticipado más arriba, sobre todo como perfección o entelequia del pensamiento.

En el Prólogo para la Historia de la filosofía de Émile Bréhier ${ }^{31}$, señala Ortega cómo Aristóteles, en su Metafisica ${ }^{32}$, opone dos conceptos decisivos: $\tau \dot{\lambda} \lambda$ o y $\pi \varepsilon ́ p \alpha \varsigma$. Hay que distinguir, dice Ortega, cuando un acto llega a plenitud teleológica y, a la vez, a su término, y cuando alcanzado el fin sigue ejerciendo su misma actividad. Aristóteles nos ofrece en 1048b 18ss la fina distinción entre movimiento y lo que, dentro de él, podemos con más rigor llamar «actividad». Al realizarse la potencia implicada en una forma en acto, esta puede destruirse y dar lugar a un cambio esencial en la sustancia. La edificación ya desaparece, junto a todas las potencias que implicaba su obra, su ěpyov, en la casa edificada, así como la bellota en la encina o la madera en el fuego. Esto Aristóteles lo llama «alteración»s sustancial. Sin embargo hay formas actuantes, sobre todo las más propias del alma, como el pensamiento, que no se agotan en la realización de sus potencias, sino, como dice espléndidamente Ortega, son potencias de sí mismas. La esencia del pensamiento es su misma potencia ( $\delta v ́ v \alpha \mu 1 \zeta)$, porque consiste en poder seguir pensando; y esa potencia, como dice a su vez espléndidamente Aristóteles, se conserva o salva en la entelequia ${ }^{33}$. De ahí se concluye que todo pensamiento concreto, realización de la esencia que es el pensar, no agota su actividad, ya que su fin es el mismo pensar, y

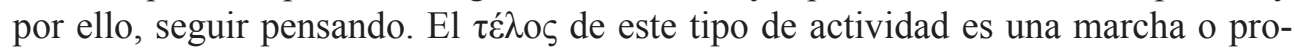
greso hacia sí misma, según las mismas reveladoras palabras que utiliza Aristóteles.

Creo que esta idea, si se considera el corpus aristotélico en relación a lo que le ha precedido y sucedido, sea quizá lo más original de su obra. Acaricia aquí, en efecto, la realidad de la vida, que es un proyecto cuyo $\tau \dot{z} \lambda o \varsigma$ se nutre de sus potencias congénitas y adquiridas, y a la vez las trasciende; que tiene potencias racionales

\footnotetext{
${ }^{31}$ Cf. Ortega y Gasset, J., Prólogo a la "Historia de la filosofía de Émile Bréhier", en O.C. VI, pp. 409-415.

${ }^{32}$ Cf. Metafisica, IX, 1048b 18,33; 1050a 17ss; en general, todo el capítulo VI del libro IX.

33 «Padecer es término que tiene más de un significado. A veces significa una destrucción ( $\varphi \theta 0 \rho \alpha ́$ ) operada por el contrario, otras, la conservación de lo que es en potencia por lo que es en entelequia

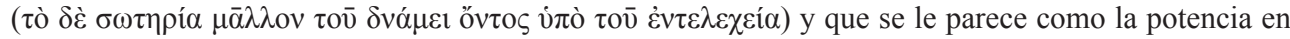
relación al acto». De Anima, II V, 417b 2-5.
} 
e irracionales según el nivel de posesión conceptual verbal que tiene sobre su misma realidad; que reobra sobre sí misma como la materia informada que, desde el proyecto actual, se vuelve a informar; que, en definitiva, al ser potencia de sí misma, implica y complica sus potencias en un progreso intrínseco, en constante búsqueda del sentido último de su realidad. Aristóteles es un fino observador empírico que, sin darse cuenta, piensa desde la estructura sistemática de su vida, describiéndola parcialmente como fenómeno natural, rehuyendo de una de sus propiedades esenciales: la historia; y, finalmente, buscando solucionar las inevitables dificultades que encuentra en el ámbito de una ontología de la naturaleza, que es, precisamente, la raíz de donde brotan las contradicciones de toda su doctrina, y que la hace, yo diría, la genial doctrina de las contradicciones.

La vida, entonces, si queremos actualizar la visión sustancialista, es una ov̉oía en el sentido de que es un patrimonio de formas, de proyectos personales que actúan como la materia -en el sentido aristotélico de «de que» ${ }^{34}$ - que está continuamente configurándose o reinterpretándose en su totalidad en cada proyecto presente, el cual, en la medida en que ya no lo es, a su vez se deposita y entra a formar parte de esa misma materia, que no deja de ser informada. Por todo ello, para concluir,

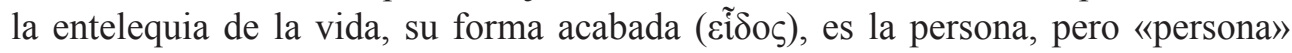

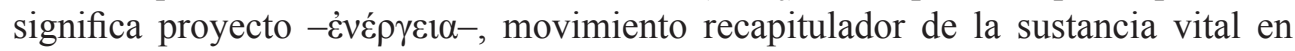
vista de una forma o figura personal siempre concreta $-\mu \rho \rho \varphi \eta ́-$, siempre nueva, que es intrínseca apertura a las demás sustancias personales, de las que se nutre y a las que se dona esencialmente.

\section{Bibliografía:}

\section{Obras de Aristóteles:}

Obras (ed. F.P. Samaranch), Madrid, Aguilar, 1967 (2 $2^{\mathrm{a}}$ edic.).

Acerca del Alma, Biblioteca Clásica Gredos, Madrid, 1978, trad. T. Calvo Martínez.

Ética a Nicómaco, Centro de Estudios Políticos y Constitucionales, Madrid, 2002, trad. de J. Marías y M. Araújo.

Ética Nicomáquea. Ética Eudemia. Madrid, Gredos. 1995, trad. J. Pallí Bonet.

Física, Consejo Superior de Investigaciones Científicas, trad. Tomás Calvo Martínez Madrid, 1996.

Política, Centro de Estudio Políticos y Constitucionales, Madrid, 1997, trad. de Julián Marías y María Araújo.

Protréptico, una exhortación a la filosofia, ABADA Editores, Madrid, 2006, "Introducción" y traducción de C. Megino Rodríguez.

${ }^{34}$ Cf. MARÍAs, J., Introducción a la filosofía, cap. VI 55. 
Metafísica, Gredos, Madrid, 1998, trad. Valentín García Yebra y también se maneja la traducción de Tomás Calvo Martínez en Biblioteca Clásica Gredos, Madrid, 2000.

Tratados de lógica (Órganon) I y II, Editorial Gredos, Madrid, 1988, trad. M. Candel Sanmartín.

Poética, Madrid, Gredos, 1999, trad. V. García Yebra.

Retórica, Clásicos Políticos, Centro de estudios constitucionales, Madrid, 1990, trad. A. Tovar.

Constitución de los atenienses, edición bilingüe, A. Bernabé, Abada, Madrid, 2005. Acerca del cielo. Meteorológicos. Madrid, Gredos,1996, M. Candel Sanmartín.

Acerca de la generación y la corrupción. Tratados breves de historia natural. Madrid, Gredos, 1987, E. La Croce, A. B. Pajares.

Investigación sobre los animales Aristóteles, Madrid, Gredos 1992, intr. C. García Gual, trad. J. Palli Bonet.

Reproducción de los animales. Madrid, Gredos (1994) Sánchez, E.

Tratados breves de historia natural. Madrid, Gredos, 1998, Pajares, A.B.

Las partes de los animales (Partes de los animales; Marcha de los animales; Movimiento de los animales), Madrid, Gredos, 2000. Intr. Trad. y notas de E. Jiménez Sánchez-Escariche y A. Alonso Miguel.

Obra biológica: De partibus animalium, De motu animalium y De Incessu Animalium, Luana, Madrid, 2010, trad. R. Bartolomé y A. Marcos.

Fragmentos. Madrid, Gredos. 2005, A. Vallejo Campos.

Otros autores citados:

De LiBerA, A., L'art des géneralités. Théories de l'abstraction, Aubier, 1999.

- La querelle des universaux. De Platon à la fin du Moyen Age. Éditions du seuil, Paris, 1996.

De Nigris, F. Libertad y Método, FUE; Madrid, 2005.

- "La razón vital de Ortega y Gasset y la analítica existencial de Heidegger", en Ideas y Valores n. 148, abril 2012.

- "El origen griego de la intencionalidad medieval", Paideia $\mathrm{n}^{\circ} 80$, sept.-dic. 2007, pp. 335-351.

- "Meditaciones filosóficas I" Cuenta y Razón, no 134, Octubre-enero 2004-2005, pp. 73-78.

- "Meditaciones filosóficas II", Cuenta y Razón, n 136, marzo-junio 2005, pp.7786

Frede, M. y Patzig, G., Metaphysik Z, Text, Übersetzung und Kommentar; Bd.I-II, Beck, Munchen, 1988. 
Guy A., Ortega y Gasset, crítico de Aristóteles. La ambiguedad del modo de pensar peripatético juzgada por el raciovitalismo. Trad. De María Luisa Pérez Torres. Madrid, Espasa Calpe (col. Austral), 1968.

Hartman, E, "Substance, Body and Soul", Aristotelian Investigations, Princeton, 1977

Heidegger, M., Phänomenologie Interpretationen zu Aristoteles (Anzeige derhermeneutischen Situation). En

Gessamtausgabe, band 62, Vittorio Klosterman, Frankfurt am Main, 2005. Trad. esp. Interpretaciones fenomenológicas sobre Aristóteles, Editorial Trotta, Madrid, 2002.

- Vom Wesen der Wahrheit, en Gessamtausgabe, band 36,37, Vittorio Klosterman, Frankfurt am Main, 2005. Trad. esp. De la esencia de la verdad. Sobre la parábola de la caverna y el Teeteto de Platón, Lecciones del semestre de invierno de 1931/32 en la Universidad de Friburgo. Editorial Herder,

Marías, J., Historia de la Filosofia, (1941) en Tomo I Obras, Revista de Occidente, Madrid, 1981.

- Introducción a la Filosofía (1947), en Tomo II de Obras, Revista de Occidente,

Madrid, 1982. Trad. it. Ragione e Vita, un'introduzione alla filosofia, Casa Editrice della Santa Croce, Roma, 2004 (Trad. F. de Nigris).

- Biografía de la Filosofía, en Tomo II de Obras, Revista de Occidente, Madrid, 1982.

- La estructura social, El Alción, colección de la Revista de Occidente, Madrid, (1955) 1972.

- Antropología Metafisica (1970), Alianza Editorial, Madrid, 1998.

- Tratado de lo Mejor, Alianza, Madrid, 1995.

- Persona, Alianza Editorial, Madrid, 1996.

Ortega y Gasset, J., Obras Completas, Revista de Occidente, 1965, vol. I-XI.

Reale, G., Guida alla lettura della “Metafisica” di Aristotele, Laterza, Roma-Bari 1997.

- Il concetto di "filosofia prima" e l'unità della metafisica di Aristotele, Vita e Pensiero, Milano 1961.

Unamuno, M, Del sentimiento trágico de la vida, Editorial Plenitud, 1966, Madrid, cap. VIII, p.149.

WitT, C, "Hylomorphism in Aristotle", Apeiron, 22, 1989, pp. 141-158.

Francesco de Nigris

Universidad Francisco de Vitoria

francescodenigris@yahoo.it 